\title{
NEW EXACT SOLUTIONS IN NON-LINEAR ELASTICITY
}

\author{
K. R. RAJAGOPAL \\ Department of Mechanical Engineering, University of Pittsburgh, Pittsburgh, PA 15261, U.S.A. \\ and \\ A. S. WINEMAN \\ Department of Mechanical Engineering and Applied Mechanics, University of Michigan, \\ Ann Arbor, MI 48109, U.S.A.
}

\begin{abstract}
In this work, we establish several new exact solutions to boundary value problems in non-linear elasticity. We show that in the case of the torsion of a slab, in addition to the classic torsion solution, there exist an infinity of solutions which are not symmetric. We study several associated boundary value problems. We also show that a nonlinearly elastic slab can exhibit nonuniform uniaxial extension solutions, in addition to the classic uniform uniaxial extension solution.
\end{abstract}

\section{INTRODUCTION}

SINCE the pioneering work of Rivlin, Green, Adkins and other co-workers in the nonlinear theory of elasticity (cf. Green and Adkins [1]) there have been few new solutions which have been exhibited. In this work, we establish solutions to several new associated boundary value problems in the theory of non-linear elasticity. An astonishing feature in all the problems dealt with is the possibility of an infinite class of exact solutions. The most interesting of the problems studied is that of the slab in pure torsion, where in addition to the classic torsion solution we exhibit the possibility of an infinity of nonsymmetric solutions. Since all the problems considered involve infinite domains, a stability analysis based on energy is not readily available. We do not study the stability problem, but leave this to a later date. The questions of stability notwithstanding, the exact solutions in themselves are so simple that they are worth recording.

Our analysis here is an outgrowth of a recent study by Rajagopal and Wineman [2], who considered the deformation of a non-linearly elastic material which is sandwiched between two infinite parallel plates, a distance ' $h$ ' apart, rotated by the same angular displacement $\Omega$ about two non-coincident axes perpendicular to the plates. The form which they assumed for the deformation corresponded to a shear (which is dependent on the coordinate normal to the plates) followed by a rigid rotation. They established exact solutions in the case of a neo-Hookean and Mooney-Rivlin material. They also studied the consequence of the non-linearly elastic material being characterized by a non-convex stored energy function. In this case they found that it is possible that the solution possess discontinuous deformation gradients and exhibit the phenomena of phase change.

In this work, we wish to study an associated, but different, class of problems. All the problems are concerned with the deformation of a slab of thickness ' $h$ ' whose other dimensions are infinite, the top and the bottom surfaces of the sandwich being bonded to rigid plates. Also, in all the following work, the non-linearly elastic material between the plates is either neo-Hookean or a Mooney-Rivlin material. First, it is our aim to investigate the consequences of rotating the top and the bottom plates by constant, but differing amounts, about non-coincident axes perpendicular to the plates (cf. Fig. 1). For the nonlinearly elastic materials in question, we are in a position to establish an infinity of exact solutions. We first exhibit exact solutions in the special case of a neo-Hookean material. It is found that a two parameter family of solutions is possible. We investigate in detail the solution which, when the offset between the axes ' $a$ ' vanishes, reduces to the classic torsion solution. However, this solution is by no means the only solution to the problem under consideration. We also compute the tractions on the upper and lower surface and find that they are not the same. It is also found that the local contribution to the resultant moment is different at the upper and lower surfaces for fixed values of the coordinates in the plane normal to the axes of the plates.

In the case of a Mooney-Rivlin material, for the kind of motion under consideration 


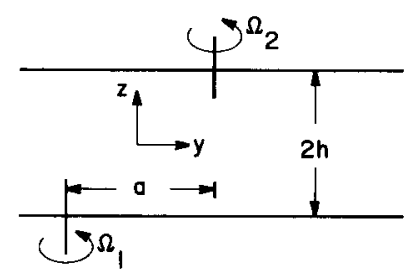

Fig. 1. Domain of deformation.

the equations of equilibrium lead to a coupled system of linear ordinary differential equations (these equations uncouple in the neo-Hookean case). The problem can be easily reduced to that of solving a single linear ordinary differential equation for a complex function. The problem is straightforward and one can determine the solution with ease. Once again an infinite class of solutions is possible.

Next, we study the problem of rotating the top and the bottom plates by different amounts about a common axis, namely the torsion problem. $\dagger$ Most interestingly, we once again exhibit an infinite one parameter family of solutions for the problem in question. The classic torsion solution belongs to this class. While it is the only symmetric solution to the problem, there are infinitely many non-symmetric solutions which are arbitrarily close to the classic torsion solution.

We also study the problem of the material being subject to finite axial stretching followed by torsion. In the case of a Mooney-Rivlin material it turns out that the equations of equilibrium simplify, after lengthy manipulations, to a coupled system of ordinary differential equations which have an amazingly simple solution. Once again, the problem exhibits the peculiar characteristic of possessing an infinity of solutions. Of course, when there is no stretching, the solutions reduce to those obtained for the pure torsion problem.

All the solutions in the case of the common axis problem and the non-coincident axes problem considered, except the last class of problems, correspond to deformations in which any plane parallel to the plates, remains in the same plane, but rotates by an amount $\Omega(z)$ which depends on the coordinate normal to the plates. That such deformations could possibly lead to non-unique solutions is motivated by Berker's investigation into the classical Newtonian fluid [3]. That a similar situation obtains in more general fluids was established by Rajagopal [4, 5] and Rajagopal and Gupta [6].

In the treatment of the uniaxial extension problem one assumes apriori that the axial stretch ratio does not vary along the direction in which the force is applied. Recently, Currie and Hayes [8] established the interesting possibility of non-uniform extension in the case of compressible non-linearly elastic materials. We present an example in which axial variation of the stretch ratio is possible in the case of incompressible non-linearly elastic materials. In the case of the neo-Hookean material it turns out that an equation which at first glance seems an exceedingly complicated non-linear ordinary differential equation possesses a simple elegant solution.

After a brief review of the necessary kinematics in the next section, we derive the appropriate form of the equations of equilibrium for the problems under consideration in Section 3. In Section 4, we study the problem of a neo-Hookean material sandwiched between two infinite parallel plates which are rotated about non-coincident axes by differing amounts. The problem and solution procedure in the case of a Mooney-Rivlin material is considered in Section 5. In the next section we study the torsion problem in the case of the neo-Hookean and the Mooney-Rivlin material. In Section 7 we study the problem of the material being subject to axial stretching followed by torsion (cf. Rajagopal and Wineman [9]). In the final section we study the non-uniform uniaxial extension of a layer.f

† We have received a personal communication from Prof. R. T. Shield [7] that the above problem and other exact solutions are being studied independently by J. M. Hill and R. T. Shield and the work is to be submitted for publication. However, at this time we have not seen the manuscript and hence do not know the exact nature of their work.

‡ It would be appropriate to point out that several interesting exact solutions have been established recently for dynamical problems in non-linear elasticity by M. M. Carroll (cf. Refs. [10-12]). 


\section{KINEMATICS}

For the problem of rotation of the plates about differing axes and a common axis, it would be natural to assume a motion of the form $\dagger$

$$
\begin{gathered}
x=[X-\bar{f}(Z)] \cos \Omega(Z)-[Y-\bar{g}(Z)] \sin \Omega(Z)+\bar{f}(Z) \\
y=[X-\bar{f}(Z)] \sin \Omega(Z)+[Y-\bar{g}(Z)] \cos \Omega(Z)+\bar{g}(Z) \\
z=Z
\end{gathered}
$$

where $X, Y, Z$ and $x, y, z$ represent the reference and the current coordinates of the same material point, respectively. The above motion represents a deformation in which material points which lie in any plane parallel to the plates continue to remain in the plane, the plane rotating about a point by an amount $\Omega(z)$. The locus of these centers of rotations is in general a curve in space passing through the centers of rotation of the top and bottom plate, the locus being defined by

$$
X=\bar{f}(Z) \quad \text { and } \quad Y=\bar{g}(Z)
$$

The representation $(2.1)_{1.2 .3}$ unfortunately leads to certain singularities in the solution. It is easy to see the physical basis for this difficulty when one considers that different angular displacements of the top and bottom plate could lead to some plane parallel to these plates which has zero rotation, in which case there are difficulties associated with the notion of the center of rotation. To avoid such difficulties, in this work we choose to express the motion in the form

$$
\begin{gathered}
x=X \cos \Omega(Z)-Y \sin \Omega(Z)+f(Z) \\
y=X \sin \Omega(Z)+Y \cos \Omega(Z)+g(Z) \\
z=Z .
\end{gathered}
$$

It follows from $(2.3)_{1,2,3}$ that the deformation gradient $F$ has the following matrix representation:

$$
\mathbf{F}=\left(\begin{array}{rrc}
C & -S & -X S \Omega^{\prime}-Y C \Omega^{\prime}+f^{\prime} \\
S & C & X C \Omega^{\prime}-Y S \Omega^{\prime}+g^{\prime} \\
0 & 0 & 1
\end{array}\right)
$$

where

$$
\begin{aligned}
& C=\cos \Omega(Z)=\cos \Omega(z) \\
& S \equiv \sin \Omega(Z)=\sin \Omega(z)
\end{aligned}
$$

and the primes denote differentiation with respect to the argument. One can now rewrite matrix (2.4) in terms of the current coordinates $x, y$, and $z$ as

$$
\mathbf{F}=\left(\begin{array}{rrc}
C & -S & -\Omega^{\prime}(y-g)+f^{\prime} \\
S & C & \Omega^{\prime}(x-f)+g^{\prime} \\
0 & 0 & 1
\end{array}\right)
$$

For the sake of simplicity let us introduce functions $\alpha(y, z)$ and $\beta(x, z)$ through

$$
\alpha(y, z)=f^{\prime}-\Omega^{\prime}(y-g)
$$

+ The above form of the motion is a generalization of the motion assumed by Rajagopal and Wineman [2] when both the top and bottom plate rotate by the same amount $\Omega$. The form of the motion assumed in Ref. [2] is the counterpart in solid mechanics of the structure assumed by Berker [3] and Rajagopal [4] in the case of fluids. 
and

$$
\beta(x, z)=g^{\prime}+\Omega^{\prime}(x-f)
$$

It follows from (2.6)-(2.8) that the left Cauchy green strain tensor $\mathbf{B}$ is given by

$$
\mathbf{B} \equiv \mathbf{F F}^{T}=\left(\begin{array}{ccc}
1+\alpha^{2} & \alpha \beta & \alpha \\
\alpha \beta & 1+\beta^{2} & \beta \\
\alpha & \beta & 1
\end{array}\right)
$$

A simple computation shows that the matrix representation for $\mathbf{B}^{-1}$ is given by

$$
\mathbf{B}^{-1}=\left(\begin{array}{rrc}
1 & 0 & -\alpha \\
0 & 1 & -\beta \\
-\alpha & -\beta & 1+\alpha^{2}+\beta^{2}
\end{array}\right) \text {. }
$$

It follows from (2.9) and (2.10) that the principal invariants of $\mathbf{B}$ are

$$
I_{\mathbf{B}} \equiv \operatorname{tr} \mathbf{B}=3+\left(\alpha^{2}+\beta^{2}\right)
$$

and

$$
\mathrm{II}_{\mathrm{B}}=3+\left(\alpha^{2}+\beta^{2}\right)
$$

The Cauchy stress $\mathbf{T}$ in the Mooney-Rivlin theory of elasticity is given by (cf. Truesdell and Noll [4])

$$
\mathbf{T}=-p \mathbf{1}+\mu\left(\frac{1}{2}+\bar{\beta}\right) \mathbf{B}-\mu\left(\frac{1}{2}-\bar{\beta}\right) \mathbf{B}^{-1}
$$

where $\mu$ and $\bar{\beta}$ are constants and $-p 1$ denotes the indeterminate spherical stress due to the constraint of incompressibility. If one requires that the strain-energy function in the Mooney-Rivlin theory be positive for all $\mathbf{B}$ it is both necessary and sufficient that (cf. Truesdell and Noll [4])

$$
\mu>0, \quad-\frac{1}{2} \leq \bar{\beta} \leq \frac{1}{2} .
$$

When $\bar{\beta}=\frac{1}{2},(2.13)$ reduces to the neo-Hookean case

$$
\mathbf{T}=-p \mathbf{1}+\mu \mathbf{B} .
$$

As we mentioned earlier, we shall first consider the neo-Hookean problem. The results for the general Mooney-Rivlin theory follows as a simple extension.

\section{EQUATIONS OF EQUILIBRIUM}

In the case of a neo-Hookean material, the equation of equilibrium

$$
\operatorname{div} \mathbf{T}+\rho \mathbf{b}=\mathbf{0}
$$

reduces to

$$
\begin{gathered}
-\frac{1}{\mu} \frac{\partial}{\partial x}(p+\rho \phi)+2 \alpha \frac{\partial \alpha}{\partial x}+\frac{\partial}{\partial y}(\alpha \beta)+\frac{\partial \alpha}{\partial z}=0 \\
-\frac{1}{\mu} \frac{\partial}{\partial y}(p+\rho \phi)+\frac{\partial}{\partial x}(\alpha \beta)+2 \beta \frac{\partial \beta}{\partial y}+\frac{\partial \beta}{\partial z}=0 \\
-\frac{1}{\mu} \frac{\partial}{\partial z}(p+\rho \phi)+\frac{\partial \alpha}{\partial x}+\frac{\partial \beta}{\partial y}=0
\end{gathered}
$$

by virtue of (2.9). In Eq. (3.1) div denotes the divergence operator, $\rho$ the density, and $b$ 
the specific body force field. In deriving (3.2)-(3.4), we have assumed that the body force field is conservative and hence derivable from a potential, i.e. $b=-\operatorname{grad} \phi$.

It follows from (2.7) and (2.8) that (3.2)-(3.4) simplify to

$$
\begin{gathered}
-\frac{1}{\mu} \frac{\partial}{\partial x}(p+\rho \phi)-\Omega^{\prime} \beta+\frac{\partial \alpha}{\partial z}=0 \\
-\frac{1}{\mu} \frac{\partial}{\partial y}(p+\rho \phi)+\Omega^{\prime} \alpha+\frac{\partial \beta}{\partial z}=0 \\
-\frac{1}{\mu} \frac{\partial}{\partial z}(p+\rho \phi)=0 .
\end{gathered}
$$

It follows from (3.5), (3.6), (2.7), and (2.8) that

$$
\frac{\partial^{2} \alpha}{\partial y \partial z}=\frac{\partial^{2} \beta}{\partial x \partial z}
$$

Equations (3.9), (2.7) and (2.8) imply that

$$
-\Omega^{\prime \prime}=\Omega^{\prime \prime}
$$

and thus

$$
\Omega(z)=\psi z+\psi_{0}
$$

where $\psi$ and $\psi_{0}$ are constants. The conditions that

$$
\Omega(-h)=\Omega_{1}
$$

and

$$
\Omega(h)=\Omega_{2}
$$

then imply that

$$
\psi_{0}=\frac{\Omega_{1}+\Omega_{2}}{2} \quad \text { and } \quad \psi=\frac{\Omega_{2}-\Omega_{1}}{2 h}
$$

Next, Eqs. (3.5)-(3.7) imply that

$$
\frac{\partial}{\partial z}\left[-\Omega^{\prime} \beta+\frac{\partial \alpha}{\partial z}\right]=0
$$

and

$$
\frac{\partial}{\partial z}\left[\Omega^{\prime} \alpha+\frac{\partial \beta}{\partial z}\right]=0
$$

It then follows from (2.7), (2.8), (3.10), (3.14), and (3.15) that $\dagger$

$$
\begin{aligned}
& f^{\prime \prime \prime}+\psi^{2} f^{\prime}=0 \\
& g^{\prime \prime \prime}+\psi^{2} g^{\prime}=0 .
\end{aligned}
$$

The boundary conditions which are appropriate for the problem of rotation of the top and bottom plates about non-coincidents axes, are (cf. Fig. 1)

$\dagger$ In our analysis we shall restrict ourselves to the case when $\psi \neq 0$. When $\psi=0, \Omega_{1}=\Omega_{2}$ and this case has been studied in detail for a much more general class of materials (materials with non-convex stored energy functions) and the Mooney material in Ref. [2]. 


$$
\begin{aligned}
& f(h)=\frac{a}{2} \sin \Omega_{2}, \quad f(-h)=-\frac{a}{2} \sin \Omega_{1} \\
& g(h)=\frac{a}{2}\left(1-\cos \Omega_{2}\right), \quad g(-h)=-\frac{a}{2}\left(1-\cos \Omega_{1}\right) .
\end{aligned}
$$

The above boundary conditions are however insufficient to determine the solutions of Eqs. (3.16) and (3.17). We now proceed to obtain the additional boundary conditions.

Integration of (3.16) and (3.17) yields

$$
\begin{aligned}
& f^{\prime \prime}+\psi^{2} f=q \\
& g^{\prime \prime}+\psi^{2} g=r
\end{aligned}
$$

where $q$ and $r$ are constant. We shall proceed to seek a solution in which $q=r=0$ which when the offset between the axes tends to zero tends to the classic torsion problem. Thus

$$
\begin{array}{r}
f^{\prime \prime}+\psi^{2} f=0 \\
g^{\prime \prime}+\psi^{2} g=0 .
\end{array}
$$

Equations (3.22) and (3.23) imply that

$$
\begin{aligned}
& f^{\prime \prime}(-h)=-\psi^{2} f(-h)=a \frac{\psi^{2}}{2} \sin \Omega_{1} \\
& g^{\prime \prime}(-h)=-\psi^{2} g(-h)=a \frac{\psi^{2}}{2}\left(1-\cos \Omega_{1}\right)
\end{aligned}
$$

In the next sub-section we shall exhibit exact solutions to (3.16) and (3.17), subject to the boundary condition (3.18), (3.19), (3.24), and (3.25).

\section{EXACT SOLUTION FOR A NEO-HOOKEAN MATERIAL IN THE CASE OF ROTATION ABOUT NON-COINCIDENT AXES}

For the sake of convenience and without loss of gencrality, we shall set $\Omega_{1}=0$ and $\Omega_{2}$ $=\Omega_{0}$. In this case it is fairly straightforward to verify that the solution to the problem under consideration is

$$
f(z)=\frac{a}{2} \sin \left[\frac{\Omega_{0}}{2}\left(\frac{z+h}{h}\right)\right]
$$

and

$$
g(z)=\frac{a}{2}\left\{\left[\operatorname{cosec} \frac{\Omega_{0}}{2}-\cos \frac{\Omega_{0}}{2} \cot \frac{\Omega_{0}}{2}\right] \sin \psi z+\sin \frac{\Omega_{0}}{2} \tan \frac{\Omega_{0}}{2} \cos \psi z\right\}
$$

where $\psi=\Omega_{0} / 2 h$. It is interesting to note that

$$
f(0)=\frac{a}{2} \sin \frac{\Omega_{0}}{2}
$$

and

$$
g(0)=\frac{a}{2} \sin \frac{\Omega_{0}}{2} \tan \frac{\Omega_{0}}{2}
$$

and hence the functions $f(z)$ and $g(z)$ do not pass through the origin. It is also worth noting that when $a=0$, the above solutions (4.1) and (4.2) reduce to the classic torsion solution. 
When $q$ and $r$ are non-zero, one could proceed in a similar manner to establish exact solutions. The functions $f(z)$ and $g(z)$ have the structure

$$
\begin{aligned}
& f(z)=A_{1} \sin \psi z+B_{1} \cos \psi z+\frac{q}{\psi^{2}} \\
& g(z)=A_{2} \sin \psi z+B_{2} \cos \psi z+\frac{r}{\psi^{2}}
\end{aligned}
$$

where the constants $A_{1}, A_{2}, B_{1}, B_{2}$ are determined from the boundary conditions. Thus, there is an infinity of solutions possible, the solutions being parameterized by $q$ and $r$. The determination of the solutions is straightforward and we shall not provide the details of the same.

Next, we determine the scalar field $p$. It follows from Eqs. (3.5)-(3.7), (3.20), and (3.21), and the definitions of $\alpha$ and $\beta$, that

$$
\begin{gathered}
-\frac{1}{\mu} \frac{\partial}{\partial x}(p+\rho \phi)-\psi^{2} x+q=0 \\
-\frac{1}{\mu} \frac{\partial}{\partial y}(p+\rho \phi)-\psi^{2} y+r=0 \\
-\frac{1}{\mu} \frac{\partial}{\partial z}(p+\rho \phi)=0 .
\end{gathered}
$$

Thus

$$
\frac{1}{\mu}(p+\rho \phi)=q x+r y-\frac{\psi^{2}}{2}\left(x^{2}+y^{2}\right)+c
$$

Hence the scalar field $p$ is independent of $z$ if $\phi$ is independent of $z$.

We now compute the tractions on the top and bottom plate associated with the above deformation. It follows from the above solutions (4.1), (4.2), and (2.15), that

$$
\begin{aligned}
\left.T_{z x}\right|_{z= \pm h} & =-\psi \mu y+\frac{a \psi \mu}{2} \\
& =\mu \frac{a}{h} \frac{\Omega_{0}}{2}\left(\frac{1}{2}-\frac{y}{a}\right) \\
\left.T_{z y}\right|_{z= \pm h} & =\psi \mu x+\frac{a \psi \mu}{2}\left(\mp \tan \frac{\Omega_{0}}{2}\right) \\
& =\mu \frac{a}{h} \frac{\Omega_{0}}{2}\left(\frac{x}{a} \mp \frac{1}{2} \tan \frac{\Omega_{0}}{2}\right) .
\end{aligned}
$$

Thus, the resultant shear $T=\left(T_{z x}^{2}+T_{z y}^{2}\right)^{1 / 2}$ on the upper and lower surface is given by

$$
T(h)=\frac{\mu a}{h} \frac{\Omega_{0}}{2}\left[\frac{r^{2}}{a^{2}}+\frac{1}{4} \sec ^{2} \frac{\Omega_{0}}{2}-\left(\frac{y}{a}+\frac{x}{a} \tan \frac{\Omega_{0}}{2}\right)\right]^{1 / 2}
$$

and

$$
T(-h)=\mu \frac{a}{h} \frac{\Omega_{0}}{2}\left[\frac{r^{2}}{a^{2}}+\frac{1}{4} \sec ^{2} \frac{\Omega_{0}}{2}+\left(\frac{x}{a} \tan \frac{\Omega_{0}}{2}-\frac{y}{a}\right)\right]^{1 / 2}
$$

Note that the resultant shear traction on the upper and lower plates differ. Also, when $a$ $=0$ 


$$
\begin{aligned}
& \left.T_{z x}\right|_{z= \pm h}=-\psi \mu y=-\frac{\mu}{2} \frac{\Omega_{0}}{h} y \\
& \left.T_{z y}\right|_{z= \pm h}=\psi \mu x=\frac{\mu}{2} \frac{\Omega_{0}}{h} x
\end{aligned}
$$

which corresponds to the 'regular torsion' problem. However, when $q \neq 0, r \neq 0$, the problem will not reduce to the regular torsion problem (cf. Section 6).

The local contribution to the resultant moment is

$$
\mathbf{M}( \pm h)=\left(x T_{z y}-y T_{z x}\right) \mathbf{k}+\left(y T_{z z} \mp h T_{z y}\right) \mathbf{i}+\left( \pm h T_{z x}-x T_{z z}\right) \mathbf{j} .
$$

Thus, if $M_{z}$ denotes the $z$-component of the local moment $\mathbf{M}$

$$
\begin{aligned}
M_{z}( \pm h) & =\left.\left(x T_{z y}-y T_{z x}\right)\right|_{ \pm h} \\
& =\mu \frac{\Omega_{0}}{h} \frac{a^{2}}{2}\left[\frac{r^{2}}{a^{2}}-\frac{1}{2}\left(\frac{y}{a} \pm \frac{x}{a} \tan \frac{\Omega_{0}}{2}\right)\right] .
\end{aligned}
$$

The first term on the right-hand side is the term corresponding to the usual torsion problem and when $a=0$, the expression reduces to the classic one. It is also interesting to note that

$$
\begin{gathered}
M_{z}(+h) \neq M_{z}(-h) \text { when } a \neq 0 . \\
\text { 5. MOONEY-RIVLIN MATERIAL }
\end{gathered}
$$

In the case of the Mooney-Rivlin material, the equations of equilibrium reduce to

$$
\begin{gathered}
-\frac{\partial}{\partial x}(p+\rho \phi)+\mu\left(\frac{1}{2}+\bar{\beta}\right)\left(-\Omega^{\prime} \beta\right)+\mu \frac{\partial \alpha}{\partial z}=0 \\
-\frac{\partial}{\partial y}(p+\rho \phi)+\mu\left(\frac{1}{2}+\bar{\beta}\right) \Omega^{\prime} \alpha+\mu \frac{\partial \beta}{\partial z}=0 \\
-\frac{\partial}{\partial z}(p+\rho \phi)-\mu\left(\frac{1}{2}-\bar{\beta}\right) \frac{\partial}{\partial z}\left(\alpha^{2}+\beta^{2}\right)=0
\end{gathered}
$$

by virtue of (2.9), (2.10), and (3.1). It follows from (5.1) and (5.2) that

$$
\frac{\partial^{2} \alpha}{\partial y \partial z}=\frac{\partial^{2} \beta}{\partial x \partial z}
$$

This in turn implies, by virtue of (2.7) and (2.8), that

$$
\Omega^{\prime \prime}=0
$$

Thus as before

$$
\Omega(z)=\psi z+\psi_{0}
$$

with $\psi_{0}=\Omega_{0} / 2, \psi=\Omega_{0} / 2 h$. Next, requiring that

$$
\frac{\partial^{2}}{\partial z \partial x}(p+\rho \phi)=\frac{\partial^{2}}{\partial x \partial z}(p+\rho \phi)
$$

implies from (5.1) and (5.3) that

$$
-\psi \mu\left(\frac{1}{2}+\bar{\beta}\right) \frac{\partial \beta}{\partial z}+\mu \frac{\partial^{2} \alpha}{\partial z^{2}}+\mu\left(\frac{1}{2}-\bar{\beta}\right) \frac{\partial}{\partial x \partial z}\left(\alpha^{2}+\beta^{2}\right)=0 .
$$


Thus

$$
\psi\left(\frac{3}{2}-3 \bar{\beta}\right) g^{\prime \prime}+f^{\prime \prime \prime}-\psi^{2}\left(\frac{1}{2}-3 \bar{\beta}\right) f^{\prime}=0 .
$$

Using Eqs. (5.2) and (5.3), one can similarly establish that

$$
\psi\left(-\frac{3}{2}+3 \bar{\beta}\right) f^{\prime \prime}+g^{\prime \prime \prime}-\psi^{2}\left(\frac{1}{2}-3 \bar{\beta}\right) g^{\prime}=0 .
$$

Note that in the case of a neo-Hookean material $\bar{\beta}=\frac{1}{2}$ and hence (5.9) and (5.10) reduce to (3.20) and (3.21), thus uncoupling the equations. In general however, the equations are a coupled system of linear ordinary differential equations.

Introducing a complex valued function $F(z)$ through

$$
F(z)=f(z)+\mathrm{i} g(z)
$$

we can re-write the coupled system (5.9) and (5.10) as

$$
F^{\prime \prime \prime}-\mathrm{i} \psi\left(\frac{3}{2}-3 \bar{\beta}\right) F^{\prime \prime}-\psi^{2}\left(\frac{1}{2}-3 \bar{\beta}\right) F^{\prime}=0 .
$$

The appropriate boundary conditions are

$$
\begin{aligned}
F(h) & =\frac{a}{2}\left[\sin \Omega_{2}+\mathrm{i}\left(1-\cos \Omega_{2}\right)\right]=\frac{a}{2}\left[\sin \Omega_{0}+i\left(1-\cos \Omega_{0}\right)\right] \\
F(-h) & =-\frac{a}{2}\left[\sin \Omega_{1}+\mathrm{i}\left(1-\cos \Omega_{1}\right)\right]=0 .
\end{aligned}
$$

As before, we can determine the additional boundary conditions by integrating (5.12). It follows from (5.12) that

$$
F^{\prime \prime}-\mathrm{i} \psi\left(\frac{3}{2}-3 \bar{\beta}\right) F^{\prime}-\psi^{2}\left(\frac{1}{2}-3 \bar{\beta}\right) F=k
$$

where $k$ is a complex constant. Again, as before, we can obtain an infinite set of solutions parameterized by $k$, to the above problem.

The solution to (5.15) is of the form

$$
F(z)=A \mathrm{e}^{s_{1} z}+B \mathrm{e}^{s^{2 z}}-\frac{k}{\psi^{2}\left(\frac{1}{2}-3 \bar{\beta}\right)}
$$

where $s_{1}$ and $s_{2}$ are given by

$$
s_{1}=\mathrm{i} \psi \quad \text { and } \quad s_{2}=\frac{\mathrm{i} \psi}{2}(1-6 \bar{\beta}) .
$$

The constants $A$ and $B$ can be obtained by using the boundary conditions (5.13) and (5.14).

\section{THE TORSION PROBLEM}

In this section we consider the classic torsion problem and show that an additional infinite class of solutions is possible for this problem. We shall consider the problem of an elastic layer of a neo-Hookean material contained between two infinite parallel plates. The top plate is rotated by an amount $\Omega_{0}$, while the bottom plate is held fixed (cf. Fig. 2 ). For the problem in question, we once again seek a motion of the form $(2.3)_{1-3}$. The well-known torsion solution corresponds to $f(Z)=0$ and $g(Z)=0$, with $\Omega(Z)=\psi_{1} z$ $+\psi_{0}$. to

In the case of a neo-Hookean material, the equations of equilibrium once again reduce 


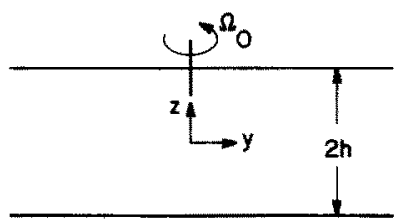

Fig. 2. Domain of deformation.

and

$$
f^{\prime \prime \prime}+\psi^{2} f^{\prime}=0
$$

$$
g^{\prime \prime \prime}+\psi^{2} g^{\prime}=0 .
$$

The appropriate boundary conditions for the problem under consideration are

$$
f(h)=0, \quad f(-h)=0, \quad g(h)=0, \quad g(-h)=0 .
$$

As before, the above boundary conditions are not sufficient to determine the solution to (6.1) and (6.2). The additional conditions which can be employed are $\dagger$

$$
f(0)=\ell, \quad g(0)=0 .
$$

While the above boundary conditions can be viewed as a kind of a normalization of the function $f(z)$, the above conditions have a physical significance when the corresponding functions $f(Z)$ and $\vec{g}(Z)$ (cf. Eq. (2.2)) are considered as defining the locus of rotations.

It is straightforward to show that when $\psi h \neq 2 n \pi$, the solution to (6.1) and (6.2), subject to (6.3) and (6.4), is

$$
\begin{aligned}
& f(z)=\frac{\ell(\cos \psi z-\cos \psi h)}{(1-\cos \psi h)} \\
& g(z) \equiv 0 .
\end{aligned}
$$

When $\ell=0$, we obtain the classic solution for the torsion problem. When $\psi h=2 n \pi$, one can easily show that $f(z)=\hat{\ell} \sin \psi z, g(z) \equiv 0$ where $\hat{\ell} \in R$. Once again, when $\ell$ $=0$, we once again obtain the torsion solution.

In the case of a Mooney-Rivlin material, the equation of equilibrium governing the torsion problem is $(5.15)$. The appropriate boundary conditions are

$$
F(h)=0, \quad F(-h)=0 \quad \text { and } \quad F(0)=\ell .
$$

\section{THE PROBLEM OF FINITE EXTENSION AND TORSION}

In this section we consider the problem of a finite extension of $\lambda$ of the infinite sandwich followed by a twisting of the top plate by an amount $\Omega$. In this case, we shall study a deformation of the following kind:

$$
\begin{aligned}
& x=\frac{1}{\sqrt{\lambda}}[X \cos \Omega(\lambda Z)-Y \sin \Omega(\lambda Z)]+f(\lambda Z) \\
& y=\frac{1}{\sqrt{ } \lambda}[X \sin \Omega(\lambda Z)+Y \cos \Omega(\lambda Z)]+g(\lambda Z) \\
& z=\lambda Z .
\end{aligned}
$$

When $\lambda=1$, the above deformation reduces to the expressions $(2.3)_{1-3}$ which we have already studied in detail. A simple computation yields the deformation gradient $\mathbf{F}$ : 


$$
\mathbf{F}=\left(\begin{array}{ccc}
\frac{C}{\sqrt{ }} & \frac{-S}{\sqrt{ } \lambda} & \sqrt{ } \lambda\left(-X S \Omega^{\prime}-Y C \Omega^{\prime}\right)+f^{\prime} \lambda \\
\frac{S}{\sqrt{ } \lambda} & \frac{C}{\sqrt{ } \lambda} & \sqrt{ } \lambda\left(X C \Omega^{\prime}-Y S \Omega^{\prime}\right)+g^{\prime} \lambda \\
0 & 0 & \lambda
\end{array}\right),
$$

where now

$$
C=\cos \Omega(\lambda Z) \quad \text { and } \quad S=\sin \Omega(\lambda Z) .
$$

We can now express $\mathbf{F}$ in terms of the present co-ordinates as

$$
\mathbf{F}=\left(\begin{array}{ccc}
\frac{C}{\sqrt{ }} & \frac{-S}{\sqrt{ } \lambda} & f^{\prime} \lambda-\Omega^{\prime} \lambda(y-g) \\
\frac{S}{\sqrt{ } \lambda} & \frac{C}{\sqrt{ } \lambda} & g^{\prime} \lambda+\Omega^{\prime} \lambda(x-f) \\
0 & 0 & \lambda
\end{array}\right) .
$$

As before, we shall define

$$
\begin{aligned}
& \alpha(y, z) \equiv f^{\prime}-\Omega^{\prime}(y-g) \\
& \beta(x, z)=g^{\prime}+\Omega^{\prime}(x-f) .
\end{aligned}
$$

Thus

$$
\mathbf{F}=\left(\begin{array}{ccc}
\frac{C}{\sqrt{\lambda}} & \frac{-S}{\sqrt{ } \lambda} & \lambda \alpha \\
\frac{S}{\sqrt{\lambda}} & \frac{C}{\sqrt{ } \lambda} & \lambda \beta \\
0 & 0 & \lambda
\end{array}\right)
$$

and the left Cauchy-Green strain tensor $\mathbf{B}$ is given by

$$
\mathbf{B}=\left(\begin{array}{ccc}
\frac{1}{\lambda}+\lambda^{2} \alpha^{2} & \lambda^{2} \alpha \beta & \lambda^{2} \alpha \\
\lambda^{2} \alpha \beta & \frac{1}{\lambda}+\lambda^{2} \beta^{2} & \lambda^{2} \beta \\
\lambda^{2} \alpha & \lambda^{2} \beta & \lambda^{2}
\end{array}\right)
$$

and

$$
\mathbf{B}^{-1}=\left(\begin{array}{ccc}
\lambda & 0 & -\lambda \alpha \\
0 & \lambda & -\lambda \beta \\
-\lambda \alpha & -\lambda \beta & \frac{1}{\lambda^{2}}+\lambda \alpha^{2}+\lambda \beta^{2}
\end{array}\right)
$$

It then follows that the first invariant is

$$
I_{B}=\frac{2}{\lambda}+\lambda^{2}\left(\alpha^{2}+\beta^{2}\right)+\lambda^{2} .
$$

It follows from the constitutive expression (2.13) for the Mooney-Rivlin material and the equations of equilibrium (3.1) that 


$$
\begin{aligned}
-\frac{\partial p}{\partial x}+\frac{\partial}{\partial x}\left[\mu\left(\frac{1}{2}+\bar{\beta}\right)\left(\frac{1}{\lambda}+\lambda^{2} \alpha^{2}\right)\right]+ & \frac{\partial}{\partial y}\left[\mu\left(\frac{1}{2}+\bar{\beta}\right) \lambda^{2} \alpha \beta\right] \\
& +\frac{\partial}{\partial z}\left[\mu\left(\frac{1}{2}+\bar{\beta}\right) \lambda^{2} \alpha+\mu\left(\frac{1}{2}-\bar{\beta}\right) \lambda \alpha\right]=0 \\
-\frac{\partial p}{\partial y}+\frac{\partial}{\partial x}\left[\mu\left(\frac{1}{2}+\bar{\beta}\right) \lambda^{2} \alpha \beta\right]+\frac{\partial}{\partial y}\left[\mu\left(\frac{1}{2}+\bar{\beta}\right)\left(\frac{1}{\lambda}+\lambda^{2} \beta^{2}\right)\right] & +\frac{\partial}{\partial z}\left[\mu\left(\frac{1}{2}+\bar{\beta}\right) \lambda^{2} \beta+\mu\left(\frac{1}{2}-\bar{\beta}\right) \lambda \beta\right]=0 \\
-\frac{\partial p}{\partial z}+\frac{\partial}{\partial x}\left[\mu\left(\frac{1}{2}+\bar{\beta}\right) \lambda^{2} \alpha+\mu\left(\frac{1}{2}-\bar{\beta}\right) \lambda \alpha\right] & +\frac{\partial}{\partial y}\left[\mu\left(\frac{1}{2}+\bar{\beta}\right) \lambda^{2} \beta\right. \\
\left.+\mu\left(\frac{1}{2}-\bar{\beta}\right) \lambda \beta\right] & -\frac{\partial}{\partial z}\left[\mu\left(\frac{1}{2}-\bar{\beta}\right)\left(\frac{1}{\lambda^{2}}+\lambda \alpha^{2}+\lambda \beta^{2}\right)\right]=0 .
\end{aligned}
$$

It follows from (7.12)-(7.14) and the definitions (7.7), and (7.7) for $\alpha(y, z)$ and $\beta(x, z)$, that

$$
\begin{gathered}
-\frac{\partial p}{\partial x}+\mu\left(\frac{1}{2}+\bar{\beta}\right) \lambda^{2} \beta\left(-\Omega^{\prime}\right)+\mu\left[\left(\frac{1}{2}+\bar{\beta}\right) \lambda^{2}+\left(\frac{1}{2}-\bar{\beta}\right) \lambda\right] \frac{\partial \alpha}{\partial z}=0 \\
-\frac{\partial p}{\partial y}+\mu\left(\frac{1}{2}+\bar{\beta}\right) \lambda^{2} \alpha \Omega^{\prime}+\mu\left[\left(\frac{1}{2}+\bar{\beta}\right) \lambda^{2}+\left(\frac{1}{2}-\bar{\beta}\right) \lambda\right] \frac{\partial \beta}{\partial z}=0 \\
-\frac{\partial p}{\partial z}-\mu\left(\frac{1}{2}-\bar{\beta}\right) \lambda \frac{\partial}{\partial z}\left(\alpha^{2}+\beta^{2}\right)=0 .
\end{gathered}
$$

It follows from Eqs. (7.15) and (7.16) that

$$
\frac{\partial^{2} \alpha}{\partial y \partial z}=\frac{\partial^{2} \beta}{\partial z \partial x}
$$

Thus, on using the definitions $(7.7)_{1}$ and $(7.7)_{2}$ for $\alpha$ and $\beta$, and (7.18)

$$
\Omega^{\prime \prime}=0 \text {. }
$$

Thus, as before

$$
\Omega(z)=\psi z+\psi_{0} .
$$

Note that the above expression for $\Omega$ is in terms of the current coordinate.

Next, it follows from (7.15) and (7.17) that

$$
-\mu\left(\frac{1}{2}+\bar{\beta}\right) \lambda^{2} \psi \frac{\partial \beta}{\partial z}+\mu\left[\left(\frac{1}{2}+\bar{\beta}\right) \lambda^{2}+\left(\frac{1}{2}-\bar{\beta}\right) \lambda\right] \frac{\partial^{2} \alpha}{\partial z^{2}}+\mu\left(\frac{1}{2}-\bar{\beta}\right) \lambda \frac{\partial^{2}}{\partial x \partial z}\left(\alpha^{2}+\beta^{2}\right)=0
$$

By virtue of $(7.7)_{1}$ and $(7.7)_{2},(7.21)$ can be expressed as

$$
\left[\frac{1}{2}\left(\lambda^{2}+\lambda\right)+\bar{\beta}\left(\lambda^{2}-\lambda\right)\right] f^{\prime \prime \prime}-\psi^{2}\left[\left(\lambda-\frac{\lambda^{2}}{2}\right)-\bar{\beta}\left(2 \lambda+\lambda^{2}\right)\right] f^{\prime}+\left(\frac{3}{2}-3 \bar{\beta}\right) \lambda \psi g^{\prime \prime}=0
$$

Similarly, (7.16) and (7.17) imply that

$$
\mu\left(\frac{1}{2}+\bar{\beta}\right) \lambda^{2} \psi \frac{\partial \alpha}{\partial z}+\mu\left[\left(\frac{1}{2}+\bar{\beta}\right) \lambda^{2}+\left(\frac{1}{2}-\bar{\beta}\right) \lambda\right] \frac{\partial^{2} \beta}{\partial z^{2}}=-\mu\left(\frac{1}{2}-\bar{\beta}\right) \lambda \frac{\partial^{2}}{\partial z \partial y}\left(\alpha^{2}+\beta^{2}\right) .
$$


Equations $(7.7)_{1}$ and $(7.7)_{2}$ now imply that

$$
\left[\frac{1}{2}\left(\lambda^{2}+\lambda\right)+\bar{\beta}\left(\lambda^{2}-\lambda\right)\right] g^{\prime \prime \prime}+\psi^{2}\left[\frac{1}{2} \lambda^{2}-\lambda+\bar{\beta}\left(\lambda^{2}+2 \lambda\right)\right] g^{\prime}+f^{\prime \prime} \psi \lambda\left(-\frac{3}{2}+3 \bar{\beta}\right)=0
$$

When $\lambda=1$, Eqs. (7.22) and (7.24) reduce to (5.9) and (5.10) for the simple torsion problem. Once again we introduce a complex valued function $F(z)$ through

$$
F(z)=f(z)+\mathrm{i} g(z)
$$

It then follows from (7.22) and (7.24) that

$$
\left[\frac{1}{2}\left(\lambda^{2}+\lambda\right)+\bar{\beta}\left(\lambda^{2}-\lambda\right)\right] F^{\prime \prime \prime}-\psi^{2}\left[\lambda-\frac{\lambda^{2}}{2}-\bar{\beta}\left(\lambda^{2}+2 \lambda\right)\right] F^{\prime}-\mathrm{i} \psi \lambda\left(\frac{3}{2}-3 \bar{\beta}\right) F^{\prime \prime}=0
$$

The appropriate boundary conditions are

$$
F(\lambda h)=0, \quad F(-\lambda h)=0
$$

and

$$
F(0)=\ell
$$

In choosing the boundary conditions (7.26) $)_{1,2}$ and the condition (7.27) we are basically choosing to interpret $x=\bar{f}(z)$ and $y=\bar{g}(z)$ as determining the locus of the centers of rotation. However, we have indicated before, there is no need to make such an interpretation (and indeed such an interpretation leads to difficulties if at a plane $z=$ const., $\Omega(z)=0)$. One could in fact replace the condition (7.27) by

$$
F(0)=k=x_{0}+\text { i } y_{0}
$$

where $k$ is a complex constant, and in this case one obtains a two parameter family of solutions. It is lengthy but straightforward to determine the solution to (7.25) subject to the boundary conditions (7.26) 1,2 and (7.27). The solution is of the form

$$
F(z)=A \mathrm{e}^{S_{12}}+B \mathrm{e}^{S_{2 z}}+C
$$

where $S_{1.2}$ are the roots of the quadratic equation

$$
\left[\frac{1}{2}\left(\lambda^{2}+\lambda\right)+\bar{\beta}\left(\lambda^{2}-\lambda\right)\right] S^{2}-\mathrm{i} \psi \lambda\left(\frac{3}{2}-3 \bar{\beta}\right) S-\psi^{2}\left[\lambda-\frac{\lambda^{2}}{2}-\bar{\beta}\left(\lambda^{2}+2 \lambda\right)\right]=0
$$

Thus

$$
S_{1,2}=\frac{i \psi \lambda\left(\frac{3}{2}-3 \bar{\beta}\right) \pm\left\{-\psi^{2} \lambda^{2}\left(\frac{3}{2}-3 \bar{\beta}\right)^{2}+4\left[\frac{1}{2}\left(\lambda^{2}+\lambda\right)+\bar{\beta}\left(\lambda^{2}-\lambda\right)\right]\right.}{\left.\times\left[\lambda-\frac{\lambda^{2}}{2}-\bar{\beta}\left(\lambda^{2}+2 \lambda\right)\right] \psi^{2}\right\}^{1 / 2}} .
$$

Interestingly, the above expression for $S_{1,2}$ simplifies to

$$
S_{1}=\mathrm{i} \psi
$$

and

$$
S_{2}=\mathrm{i} \psi \lambda\left[\frac{2-\lambda-2 \bar{\beta}(2+\lambda)}{\lambda^{2}+\lambda+2 \bar{\beta}\left(\lambda^{2}-\lambda\right)}\right]
$$


It then follows that

$$
f(\lambda Z)=\frac{\ell}{\bar{\Delta}}[\sin \psi h \lambda M \cos \psi \lambda Z-\sin \psi \lambda h \cos \psi M \lambda Z+\Delta]
$$

and

$$
g(\lambda Z)=\frac{\ell}{\bar{\Delta}}[\sin \psi h \lambda M \sin \psi \lambda Z-\sin \psi \lambda h \sin \psi M \lambda Z]
$$

where

$$
\Delta=\sin \psi h \lambda(1-M), \quad M=\frac{2-\lambda-2 \bar{\beta}(2+\lambda)}{1+\lambda+2 \bar{\beta}(\lambda-1)}
$$

and

$$
\bar{\Delta}=4 \sin \psi h \lambda \frac{(1-M)}{2} \sin \frac{\psi h \lambda}{2} \sin \frac{\psi h \lambda M}{2} .
$$

We conclude this section by studying the problem of the infinite sandwich being extended by a finite amount and then being twisted by $\Omega_{1}$ and $\Omega_{2}$ about non-coincident axes. For this problem, we once again seek a deformation of form (7.1)-(7.3). Thus we obtain the same equilibrium Eq. (7.25). The only difference is in the boundary condition, and for the above problem, the boundary conditions are (for the sake of convenience we shall set $\Omega_{1}=0$ and $\Omega_{2}=\Omega_{0}$ )

$$
\begin{aligned}
f(\lambda h) & =\frac{a}{2 \sqrt{ } \lambda} \sin \Omega_{2}=\frac{a}{2 \sqrt{ } \lambda} \sin \Omega_{0} \\
f(-\lambda h) & =-\frac{a}{2 \sqrt{ } \lambda} \sin \Omega_{1}=0 \\
g(\lambda h) & =\frac{a}{2 \sqrt{ } \lambda}\left(1-\cos \Omega_{2}\right)=\frac{a}{2 \sqrt{ } \lambda}\left(1-\cos \Omega_{0}\right)
\end{aligned}
$$

and

$$
g(-\lambda h)=\frac{a}{2 \sqrt{\lambda}}\left(1-\cos \Omega_{1}\right)=0
$$

Thus

$$
\begin{aligned}
F(\lambda h) & =\frac{a}{2 \sqrt{ } \lambda}\left[\sin \Omega_{0}+\mathrm{i}\left(1-\cos \Omega_{0}\right)\right] \\
F(-\lambda h) & =0 .
\end{aligned}
$$

Since $\Omega_{1}=0$ and $\Omega_{2}=\Omega_{0}$, it follows that

Then

$$
\psi=\frac{\Omega_{0}}{2 \lambda h} .
$$

$$
\begin{aligned}
F=\frac{a}{4 \delta} \sqrt{ } & {\left[\mathrm{e}^{\mathrm{i}\left(\left(\Omega_{0} / 2\right)(z / h)+\Omega_{0}-\left(\Omega_{0} / 2\right) M\right]}-\mathrm{e}^{\mathrm{i}\left[\left(\Omega_{0} / 2\right)(z / h)-\left(\Omega_{0} / 2\right) M\right]}+\mathrm{e}^{\mathrm{i}\left[\left(\Omega_{0} / 2\right) M(z / h)-\left(\Omega_{0} / 2\right)\right]}\right.} \\
& -\mathrm{e}^{\mathrm{i}\left[\left(\Omega_{0} / 2\right) M(z / h)+\left(\Omega_{0} / 2\right)\right]}+A_{3}\left[\begin{array}{c}
\sin \frac{\Omega_{0} M}{2} \mathrm{e}^{\mathrm{i}\left(\Omega_{0} / 2\right)(z / h)}-\sin \frac{\Omega_{0}}{2} \mathrm{e}^{\mathrm{i}\left(\Omega_{0} / 2\right) M(z / h)} \\
\delta
\end{array}\right]
\end{aligned}
$$


where

$$
\delta=\sin (\psi M \lambda h-\psi \lambda h)=\sin \frac{\Omega_{0}}{2}(M-1)
$$

This then implies that

$$
\begin{aligned}
& f(\lambda Z)=\frac{a}{4 \delta \sqrt{ } \lambda}\left[\cos \left(\frac{\Omega_{0}}{2} \frac{z}{h}+\Omega_{0}-\frac{\Omega_{0}}{2} M\right)-\cos \left(\frac{\Omega_{0}}{2} \frac{z}{h}-\frac{\Omega_{0}}{2} M\right)+\cos \left(\frac{\Omega_{0}}{2} M \frac{z}{h}-\frac{\Omega_{0}}{2}\right)\right. \\
& \left.-\cos \left(\frac{\Omega_{0}}{2} M \frac{z}{h}+\frac{\Omega_{0}}{2}\right)\right]+A_{3}\left[1-\frac{\sin \frac{\Omega_{0} M}{2} \cos \frac{\Omega_{0}}{2} \frac{z}{h}-\sin \frac{\Omega_{0}}{2} \cos \frac{\Omega_{0}}{2} M \frac{z}{h}}{\Delta}\right]
\end{aligned}
$$

and

$$
\begin{aligned}
& g(\lambda Z)=\frac{a}{4 \delta \sqrt{ } \lambda}\left[\sin \left(\frac{\Omega_{0}}{2} \frac{z}{h}+\Omega_{0}-\frac{\Omega_{0}}{2} M\right)-\sin \left(\frac{\Omega_{0}}{2} \frac{z}{h}-\frac{\Omega_{0} M}{2}\right)\right. \\
& \left.+\sin \left(\frac{\Omega_{0}}{2} M \frac{z}{h}-\frac{\Omega_{0}}{2}\right)-\sin \left(\frac{\Omega_{0}}{2} M \frac{z}{h}+\frac{\Omega_{0}}{2}\right)\right] \\
& -\frac{A_{3}}{\Delta}\left(\sin \frac{\Omega_{0} M}{2} \sin \frac{\Omega_{0}}{2} \frac{z}{h}-\sin \frac{\Omega_{0}}{2} \sin \frac{\Omega_{0}}{2} M \frac{z}{h}\right) .
\end{aligned}
$$

Note that the above yield a one parameter family of solutions in terms of the constant $A_{3}$, and this is to be expected, as we have not imposed an additional condition as before, e.g. the location where the locus of the centers of the rotations cuts the $z=0$ plane.

\section{NON-UNIFORM UNIAXIAL EXTENSION}

Consider an infinite layer of an incompressible nonlinear elastic material. Let $\mathbf{X}=(X$, $Y, Z)$ denote the position of the particle in its reference configuration and let $\mathbf{x}=(x, y$, $z$ ) denote the position of the particle in its current configuration. Let the layer be bounded by the planes $Z=h$ and $Z=0$. Consider the deformation

$$
x=\frac{1}{\sqrt{ }\left(\lambda^{\prime}(Z)\right)} X, \quad y=\frac{1}{\left.\sqrt{(} \lambda^{\prime}(Z)\right)} Y, \quad z=\lambda(Z)
$$

where prime denotes differentiation with respect to the argument. In the usual assumption for uni-axial extension $\lambda(Z)=\lambda Z$, where $\lambda$ is a constant. The deformation gradient $F$ is given by

$$
\mathbf{F}=\left(\begin{array}{ccc}
\frac{1}{\sqrt{\lambda^{\prime}}} & 0 & \alpha \\
0 & \frac{1}{\sqrt{\lambda^{\prime}}} & \beta \\
0 & 0 & \lambda^{\prime}
\end{array}\right)
$$

where

$$
\alpha=-\frac{1}{2}\left(\lambda^{\prime}\right)^{-3 / 2} \lambda^{\prime \prime} X
$$

and

$$
\beta=-\frac{1}{2}\left(\lambda^{\prime}\right)^{-3 / 2} \lambda^{\prime \prime} Y
$$


Thus, the Cauchy-Green strain tensor $\mathbf{B}=\mathbf{F F}^{T}$ has the following matrix representation

$$
\mathbf{B}=\left(\begin{array}{ccc}
\frac{1}{\lambda^{\prime}}+\alpha^{2} & \alpha \beta & \alpha \lambda^{\prime} \\
\alpha \beta & \frac{1}{\lambda^{\prime}}+\beta^{2} & \beta \lambda^{\prime} \\
\alpha \lambda^{\prime} & \beta \lambda^{\prime} & \lambda^{\prime 2}
\end{array}\right) .
$$

Also, the tensor $F^{-1}$ has the form

$$
\mathbf{F}^{-1}=\left(\begin{array}{ccc}
\sqrt{\lambda^{\prime}} & 0 & -\frac{\alpha}{\sqrt{\lambda^{\prime}}} \\
0 & \sqrt{\lambda^{\prime}} & -\frac{\beta}{\sqrt{\lambda^{\prime}}} \\
0 & 0 & \frac{1}{\lambda^{\prime}}
\end{array}\right)
$$

For the purposes of illustration of the possibility of non-uniform solutions for the stretch, we shall assume that the nonlinearly elastic material is neo-Hookean. The Cauchy stress $\mathbf{T}$ in such a material is given by (2.15).

In view of the assumed form of the deformation, we find that it is computationally more convenient to express the equations of equilibrium in terms of the reference configuration. In the absence of body forces, the equations of equilibrium take the form

$$
\frac{\partial T_{i j}}{\partial x_{j}}=\frac{\partial T_{i j}}{\partial X_{p}} \frac{\partial X_{p}}{\partial x_{j}}=\frac{\partial T_{i j}}{\partial X_{p}} F_{p j}^{-1}=0
$$

A straightforward but lengthy calculation yields the following equations of equilibrium (here we have replaced $p / \mu$ by $p$ for convenience):

$$
\begin{gathered}
-\sqrt{ } \lambda^{\prime} \frac{\partial p}{\partial X}+\frac{X}{\lambda^{\prime}} \frac{\mathrm{d}}{\mathrm{d} Z}\left(-\frac{1}{2} \lambda^{\prime \prime}(\lambda)^{-1 / 2}\right)+\frac{\left(\lambda^{\prime \prime}\right)^{2} X}{2\left(\lambda^{\prime}\right)^{5 / 2}}=0 \\
-\sqrt{ } \lambda^{\prime} \frac{\partial p}{\partial Y}+\frac{Y}{\lambda^{\prime}} \frac{\mathrm{d}}{\mathrm{d} Z}\left(-\frac{1}{2} \lambda^{\prime \prime}\left(\lambda^{\prime}\right)^{1 / 2}\right)+\frac{\left(\lambda^{\prime \prime}\right)^{2} Y}{2\left(\lambda^{\prime}\right)^{5 / 2}}=0 \\
\lambda^{\prime \prime}-\frac{1}{\lambda^{\prime}} \frac{\partial p}{\partial Z}-\frac{\lambda^{\prime \prime}}{2\left(\lambda^{\prime}\right)^{2}}\left(X \frac{\partial p}{\partial X}+Y \frac{\partial p}{\partial Y}\right)=0
\end{gathered}
$$

Let us define

$$
f(Z)=\frac{1}{\left(\lambda^{\prime}\right)^{3 / 2}} \frac{\mathrm{d}}{\mathrm{d} Z}\left(-\frac{1}{2} \lambda^{\prime \prime}\left(\lambda^{\prime}\right)^{-1 / 2}\right)+\frac{\left(\lambda^{\prime \prime}\right)^{2}}{2\left(\lambda^{\prime}\right)^{3}}
$$

Then

$$
\begin{aligned}
& \frac{\partial p}{\partial X}=X f(Z) \\
& \frac{\partial p}{\partial Y}=Y f(Z)
\end{aligned}
$$

and

$$
\frac{\partial p}{\partial Z}=\lambda \lambda^{\prime \prime}-\frac{\lambda^{\prime \prime}}{2 \lambda^{\prime}}\left(X^{2}+Y^{2}\right) f(Z) .
$$


We eliminate the scalar $p$ by the standard procedure of cross-differentiation to find that

$$
\frac{\mathrm{d} f}{\mathrm{~d} Z}=-\frac{\lambda^{\prime \prime}}{\lambda^{\prime}} f(Z)
$$

Thus, a simple integration yields

$$
\lambda^{\prime} f=\text { const. }=C_{1} .
$$

It follows from (8.11) and (8.16) that

$$
\lambda^{\prime \prime \prime}-\frac{3}{2} \frac{\left(\lambda^{\prime \prime}\right)^{2}}{\lambda^{\prime}}=C \lambda^{\prime}
$$

where

$$
C=-2 C_{1}
$$

Let

$$
\lambda^{\prime}(Z) \equiv a(Z)
$$

then

$$
a^{\prime \prime}-\frac{3}{2} \frac{a^{\prime 2}}{a}=C a
$$

which reduces to the form

$$
\frac{\mathrm{d}}{\mathrm{d} Z}\left(a^{-3 / 2} a^{\prime}\right)=C a^{-1 / 2}
$$

It follows that

$$
\frac{\mathrm{d}^{2}}{\mathrm{~d} Z^{2}}\left(a^{-1 / 2}\right)+\frac{C}{2} a^{-1 / 2}=0 .
$$

Thus, when $C>0$,

$$
a^{-1 / 2}=\left(\lambda^{\prime}\right)^{-1 / 2}=A_{1} \sin V /\left(\frac{C}{2}\right) Z+B_{1} \cos V /\left(\frac{C}{2}\right) Z
$$

and

$$
\lambda^{\prime}(Z)=\frac{1}{\left\{A_{1} \sin \sqrt{ }\left(\frac{C}{2}\right) Z+B_{1} \cos V /\left(\frac{C}{2}\right) Z\right\}^{2}}
$$

Let us consider the case of a layer which is of thickness $H$, fixed at the bottom. The appropriate conditions at the bottom and top layer would be

$$
\begin{array}{r}
z(0)=0 \\
z(H)=h
\end{array}
$$

where the coordinate $Z=H$ is at present at the coordinate $z=h$. Let us define a function $g\left(A_{1}, B_{1}, C, Z\right)$ through

$$
\lambda^{\prime}(Z) \equiv g\left(A_{1}, B_{1}, C, Z\right)
$$

Then

$$
z(Z)=\int_{0}^{Z} g\left(A_{1}, B_{1}, C, \bar{Z}\right) \mathrm{d} \bar{Z}
$$


Equations (8.26) and (8.28) define a relationship between the constants $A_{1}, B_{1}$, and $C$. A restriction on the possible values of $A_{1}, B_{1}$, and $C$ arises from the fact that $\lambda^{\prime}(Z)$ be bounded.

When $C<0$, then eqn (8.22) exhibits solutions of the form

$$
a^{-1 / 2}=\left(\lambda^{\prime}\right)^{-1 / 2}=A_{2} e^{\sqrt{\frac{C}{2}} z}+B_{2} e^{-\sqrt{\frac{C}{2}} z}
$$

and

$$
\lambda^{\prime}=\frac{1}{\left\{A_{2} e^{\sqrt{\frac{c}{2}} z}+B_{2} e^{\sqrt{\frac{c}{2} z}}\right\}^{1 / 2}} .
$$

Once again the comments following eqn (8.24) apply in this case.

Note that, when $C=0$

$$
\lambda^{\prime}(Z)=\text { const. }
$$

which corresponds to the classical homogeneous solution. However, as we have seen this is by no means the only solution. In fact, there exists a one parameter family (say $C$ ) of solutions to the problem. By appropriate choices of the constants, one can make $\lambda^{\prime}(Z)$ as close to the classical constant solution, as desired.

Acknowledgement-K. R. Rajagopal would like to acknowledge the solid mechanics program of the National Science Foundation and the Mathematics Research Center at the University of Wisconsin, Madison for their support.

\section{REFERENCES}

[1] A. E. GREEN and J. E. ADKINS, Large Elastic Deformations. Oxford (1970).

[2] K. R. RAJAGOPAL and A. S. WINEMAN, J. Struct. Mech. 12, 471 (1984).

[3] R. BERKER, Archiwum Mech. Stosow. 31, 265 (1979).

[4] K. R. RAJAGOPAL, Arch. Rat. Mech. Analysis 79, 39 (1982).

[5] K. R. RAJAGOPAL, J. Non-Newtonian Fluid Mech. 9, 185 (1981).

[6] K. R. RAJAGOPAL and A. S. GUPTA, Int. J. Engng Sci. 19, 1401 (1981).

[7] R. T. SHIELD, Private communication, (1984).

[8] P. K. CURRIE and M. HAYES, Proc. IUTAM Symp. on Finite Elasticity (Lehigh University, 1980) (Edited by D. E. Carlson and R. T. Shield), pp. 142-150. Martinus Nijhoff, Hague, Netherlands (1982).

[9] K. R. RAJAGOPAL and A. S. WINEMAN, University of Wisconsin, Math. Research Center Report (1983).

[10] M. M. CARROLL, Q. J. Mech. Appl. Math 30, 223 (1976)

[11] M. M. CARROLL, J. Appi. Math. Phys. 25, 83 (1974).

[12] M. M. CARROLL, J. Elast. 8, 323 (1978). 